\title{
活性位高度暴露的钴/氮/碳电催化剂的构建及氧还原性能研究
}

\author{
张志琦 \\ 葛承宣陈玉刚吴强* \\ 杨立军 \\ 王喜章 \\ 胡征
}

(介观化学教育部重点实验室 南京大学化学化工学院 南京 210023)

\begin{abstract}
摘要 金属/氮/碳催化剂( $\mathrm{M} / \mathrm{N} / \mathrm{C}, \mathrm{M}=\mathrm{Fe} 、 \mathrm{Co}$ 等)是最有发展前景的非贵金属电催化剂之一，其性能依赖于催化剂表面 的活性物种密度. 通过常规的热解含氮前驱物与金属盐的方法制得的催化剂往往存在金属活性物种被包埋而不能有效 利用的缺点. 考虑到石墨相氮化碳 $\left(\mathrm{g}-\mathrm{C}_{3} \mathrm{~N}_{4}\right)$ 富含类吡啶氮和亚纳米孔腔结构, 将 $\mathrm{g}_{-} \mathrm{C}_{3} \mathrm{~N}_{4}$ 包覆在高导电性碳纳米笼 (hCNC) 表面, 进而利用表层 $\mathrm{g}-\mathrm{C}_{3} \mathrm{~N}_{4}$ 的配位和限域作用针定大量 $\mathrm{Co}^{2+}$ 离子, 获得的 $\mathrm{Co} / \mathrm{g}-\mathrm{C}_{3} \mathrm{~N}_{4} / \mathrm{hCNC}$ 复合物经热解后形 成了活性位高度暴露、导电性好、孔结构丰富的 $\mathrm{Co} / \mathrm{N} / \mathrm{C}$ 催化剂. $800{ }^{\circ} \mathrm{C}$ 热解得到的最优化催化剂在碱性介质中展现出 优异氧还原活性, 其起始电位 $(0.97 \mathrm{~V})$ 与商业 $\mathrm{Pt} / \mathrm{C}$ 催化剂相当, 且抗甲醇干扰性能和稳定性优异. 此项研究提供了一种 构建具有高度暴露活性位的 $\mathrm{M} / \mathrm{N} / \mathrm{C}$ 催化剂的有效策略.

关键词 钴/氮/碳; 氧还原催化剂; 高度暴露活性位; 碳纳米笼; 燃料电池
\end{abstract}

\section{Construction of Cobalt/Nitrogen/Carbon Electrocatalysts with Highly Exposed Active Sites for Oxygen Reduction Reaction}

\author{
Zhang, Zhiqi Ge, Chengxuan Chen, Yugang Wu, Qiang* Yang, Lijun \\ Wang, Xizhang $\mathrm{Hu}$, Zheng \\ (Key Laboratory of Mesoscopic Chemistry of MOE, School of Chemistry and Chemical Engineering, Nanjing University, \\ Nanjing 210023, China)
}

\begin{abstract}
The ever-growing crises of fossil fuel shortage and environmental pollution urgently call for the exploration of clean and renewable energies. Fuel cells present high power efficiency and emit zero pollutants, showing great potential in the future energy system. The main bottleneck of fuel cell commercialization is the sluggish oxygen reduction reaction (ORR) at the cathode. To date, the most active electrocatalysts for ORR are platinum and its alloys. However, the scarcity, high cost and susceptibility to methanol crossover of precious metals hinder the large-scale application of fuel cells. The development of highly efficient and stable non-precious metal ORR electrocatalysts with high resistance to methanol crossover is of great significance. $\mathrm{M} / \mathrm{N} / \mathrm{C}(\mathrm{M}=\mathrm{Fe}, \mathrm{Co}$, etc. $)$ catalysts are attractive non-precious metal based ORR electrocatalysts and their performance depends on the density of active sites on the catalyst surface. The common synthesis of M/N/C catalysts is to pyrolyze the mixture of metal salt, nitrogen-containing precursor and carbon support. However, so-synthesized catalysts usually contain large metal-based particles, leading to the shortcomings of low density and partial embedding of active sites. Graphitic carbon nitride $\left(\mathrm{g}-\mathrm{C}_{3} \mathrm{~N}_{4}\right)$ with high concentration of pyridine-like nitrogen in heptazine heterorings can provide abundant and uniform nitrogen coordination sites, which can capture metal ions by the interaction between metal ions and $\mathrm{N}$ sites. In addition, $\mathrm{g}-\mathrm{C}_{3} \mathrm{~N}_{4}$ would be decomposed largely during pyrolysis, which is beneficial to form highly exposed M/N/C active sites by pyrolyzing the $\mathrm{g}_{-} \mathrm{C}_{3} \mathrm{~N}_{4}$ with adsorbed metal ions. Herein, we reported the construction of $\mathrm{Co} / \mathrm{N} / \mathrm{C}$ electrocatalysts with highly exposed active sites. Specifically, the g- $\mathrm{C}_{3} \mathrm{~N}_{4}$ was uniformly supported on the surface of high-conductive hierarchical carbon nanocages (hCNC) by the impregnation and pyrolysis process, leading to the formation of $\mathrm{g}_{-} \mathrm{C}_{3} \mathrm{~N}_{4} / \mathrm{hCNC}$ composite. $\mathrm{Co}^{2+}$ ions were then captured by the $\mathrm{g}-\mathrm{C}_{3} \mathrm{~N}_{4}$ species on the surface owing to the interaction between the lone pair electrons of nitrogen and the $\mathrm{Co}^{2+}$ ions, and the subsequent pyrolysis led to the $\mathrm{Co} / \mathrm{N} / \mathrm{C}$ catalysts with highly exposed active sites, high conductivity and multiscale pore structure. The optimized catalyst obtained at $800{ }^{\circ} \mathrm{C}$ exhibits excellent ORR performance in alkaline medium, with a high onset potential $(0.97 \mathrm{~V})$ comparable to commercial $\mathrm{Pt} / \mathrm{C}$ catalyst, while much better stability and high immunity to methanol crossover. This study demonstrates an effective strategy for the construction of high-efficient $\mathrm{M} / \mathrm{N} / \mathrm{C}$ catalysts with highly exposed active sites.
\end{abstract}

Keywords cobalt/nitrogen/carbon; oxygen reduction electrocatalysts; highly exposed active sites; carbon nanocages; fuel cell

\footnotetext{
* E-mail: wqchem@nju.edu.cn; Tel. \& Fax: 025-89683696

Received August 7, 2018; published November 7, 2018.

Supporting information for this article is available free of charge via the Internet at http://sioc-journal.cn.

Project supported by the National Key Research and Development Program of China (No. 2017YFA0206500), National Natural Science Foundation of China (Nos. 21773111, 21473089, 51571110, 21573107), Changzhou Technology Support Program (No. CE20130032), Priority Academic Program Development of Jiangsu Higher Education Institution, and Fundamental Research Funds for the Central Universities.

项目受国家重点研发计划(No. 2017YFA0206500)、国家自然科学基金(Nos. 21773111，21473089，51571110，21573107)、常州市科技计划(No. CE20130032)、江苏高校优势学科建设工程资助项目和中央高校基本科研业务费专项资金资助.
} 


\section{1 引言}

燃料电池具有清洁、高效等特点, 是国家重点发展 的战略领域之一. 制约燃料电池大规模应用的瓶颈之一 是阴极氧还原反应 $(\mathrm{ORR})$ 的动力学过程缓慢 ${ }^{[1]}$, 需要使 用高效电催化剂, 而目前使用的是昂贵的 Pt 基催化 剂 ${ }^{[2,3]}$. 众所周知, Pt 的资源稀缺, 且 Pt 基催化剂的 ORR 稳定性较差, 容易受到甲醇渗透等问题的困扰 ${ }^{[3,4]}$. 开发 高效稳定、具有良好抗甲醇渗透能力的非贵金属 ORR 电催化剂具有重要意义 ${ }^{[5]}$. 据此, 价格低廉的 $M / N / C$ 催 化剂( $M=F e 、 C o$ 等) 是备受关注的非贵金属 ORR 电催 化剂, 其性能依赖于催化剂表面的活性物种密度. 文献 中常采用热解金属盐、含氮前驱物(如聚苯胺、聚吡咯、 双氰胺等)与碳载体的方法制备 $\mathrm{M} / \mathrm{N} / \mathrm{C}$ 催化剂 ${ }^{[6-9]}$. 研究 发现, M/N/C 催化剂的理想载体应具备大比表面积、丰 富孔结构及高导电性等特征 ${ }^{[10,11]}$; 含氮前驱物应当具有 高氮含量, 热解后产生的氮物种要均匀分布在催化剂表 面 ${ }^{[7]}$, 与电解液接触良好. 但是, 通过这种方法制得的 产物中往往存在大量金属颗粒 (大多为 $5 \sim 15 \mathrm{~nm}$ ), 导致 活性位密度低、金属活性位被包埋等不足, 严重影响其 ORR 活性 ${ }^{[7,9]}$.

石墨化 $\mathrm{C}_{3} \mathrm{~N}_{4}\left(\mathrm{~g}-\mathrm{C}_{3} \mathrm{~N}_{4}\right)$ 由周期有序的庚嗪环单元构 成, 富含类吡啶氮且具有亚纳米孔腔结构, 可利用分布 均匀的类吡啶氮原子与金属离子的配位作用实现金属 物种的高度分散 ${ }^{[12,13]}$, 且可通过亚纳米孔腔的物理限域 作用阻止金属物种的团聚 ${ }^{[14,15]}$. 此外, 热解过程中绝大 部分的 $\mathrm{g}-\mathrm{C}_{3} \mathrm{~N}_{4}$ 发生分解, 从而使形成的 $\mathrm{M} / \mathrm{N} / \mathrm{C}$ 活性位 高度暴露, 减少活性位被包埋的现象. 利用 $\mathrm{g}-\mathrm{C}_{3} \mathrm{~N}_{4}$ 中氮 原子的配位作用和孔腔限域作用已成功构建了贵金属 单原子催化剂, 有效提高了光催化制氢活性 ${ }^{[16,17]}$. 但是, g- $\mathrm{C}_{3} \mathrm{~N}_{4}$ 及其热分解产物的导电性差, 限制了其电催化性 能 ${ }^{[18,19]}$. 最近, Zhang 等将 $\mathrm{g}-\mathrm{C}_{3} \mathrm{~N}_{4}$ 包裹于石墨烯内改善 导电性, 在电解水析氢及氧还原反应中展现出一定的催 化活性 ${ }^{[20 ~ 23]}$, 但是石墨烯的 $\pi-\pi$ 堆垛容易导致部分活性
位的包埋, 降低其电催化性能. 我们课题组开发了导电 性好、比表面积大、孔结构丰富的 3D 分级结构碳基纳 米笼 $(\mathrm{hCNC})$, 可有效避免类似石墨烯的 $\pi-\pi$ 堆垛, 在能 源存储和转化中展现出优异性能 ${ }^{[24 ~ 28]}$. 本研究中, 将 $\mathrm{g}-\mathrm{C}_{3} \mathrm{~N}_{4}$ 原位生长在高导电性 $\mathrm{hCNC}$ 表面, 进而利用表层 $\mathrm{g}-\mathrm{C}_{3} \mathrm{~N}_{4}$ 的配位和限域作用针定大量 $\mathrm{Co}^{2+}$ 离子, 热解 $\mathrm{Co}-\mathrm{g}-\mathrm{C}_{3} \mathrm{~N}_{4} / \mathrm{hCNC}$ 复合物制备了活性位高度暴露的 $\mathrm{Co} / \mathrm{N} / \mathrm{C}$ 催化剂. 研究了该催化剂在碱性介质中的 ORR 活性、稳定性和抗甲醇性能, 展示了一种构建具有高度 暴露活性位的 $\mathrm{M} / \mathrm{N} / \mathrm{C}$ 催化剂的有效策略.

\section{2 结果与讨论}

图 1 是 $\mathrm{Co} / \mathrm{N} / \mathrm{C}$ 催化剂的制备路线图. 采用原位 $\mathrm{MgO}$ 模板法制备 $\mathrm{hCNC}$ 载体, 它是由空心纳米笼相互连 接形成纳米片, 进而由纳米片组装而成的多孔微米 球 ${ }^{[24]}$, 其比表面积为 $1450 \mathrm{~m}^{2} \cdot \mathrm{g}^{-1}$, 体相电导率为 $395 \mathrm{~S} \cdot$ $\mathrm{m}^{-1}$. 通过浸渍法将尿素和 $\mathrm{hCNC}$ 混合均匀, 在 $550{ }^{\circ} \mathrm{C}$ 热解制得了 $\mathrm{g}-\mathrm{C}_{3} \mathrm{~N}_{4}$ 包覆的 $\mathrm{g}-\mathrm{C}_{3} \mathrm{~N}_{4} / \mathrm{hCNC}$ 复合物; 将此 复合物分散于含 $\mathrm{Co}^{2+}$ 离子的溶液中, 通过氮位孤对电 子与金属离子的配位作用，将 $\mathrm{Co}^{2+}$ 选择性吸附在复合 物表面 ${ }^{[29,30]}$, 多余的 $\mathrm{Co}^{2+}$ 通过洗涤除去, 以避免其在后 续热解过程中发生团聚; 获得的 Co-g- $\mathrm{C}_{3} \mathrm{~N}_{4} / \mathrm{hCNC}$ 复合 物经高温热解转化为 $\mathrm{Co} / \mathrm{N} / \mathrm{C}$ 催化剂. 由于 $\mathrm{Co}-\mathrm{g}-\mathrm{C}_{3} \mathrm{~N}_{4} /$ $\mathrm{hCNC}$ 中 $\mathrm{Co}^{2+}$ 与氮原子配位相连, 且大部分的 $\mathrm{g}_{-} \mathrm{C}_{3} \mathrm{~N}_{4}$ 在热解中分解, 导致 $\mathrm{Co} / \mathrm{N} / \mathrm{C}$ 活性位高度暴露且均匀分 散于 $\mathrm{hCNC}$ 载体表面, $\mathrm{Co}$ 物种团聚和包埋的情形得到极 大的抑制.

图 2 为中间产物的形貌及结构表征. g- $\mathrm{C}_{3} \mathrm{~N}_{4} / \mathrm{hCNC}$ 复合物保留了 $\mathrm{hCNC}$ 的分级多孔结构(图 S1a), 相应的 透射电镜(TEM)照片显示在纳米笼表面较均匀地附着一 些纳米颗粒(图 2a，2b), 没有观察到大块片状结构 $\mathrm{g}-\mathrm{C}_{3} \mathrm{~N}_{4}$ (图 S1b), 表明 $\mathrm{g}-\mathrm{C}_{3} \mathrm{~N}_{4}$ 高度分散在 $\mathrm{hCNC}$ 表面. $\mathrm{X}$ 射线衍射(XRD)谱显示 $\mathrm{g}-\mathrm{C}_{3} \mathrm{~N}_{4} / \mathrm{hCNC}$ 复合物具有与

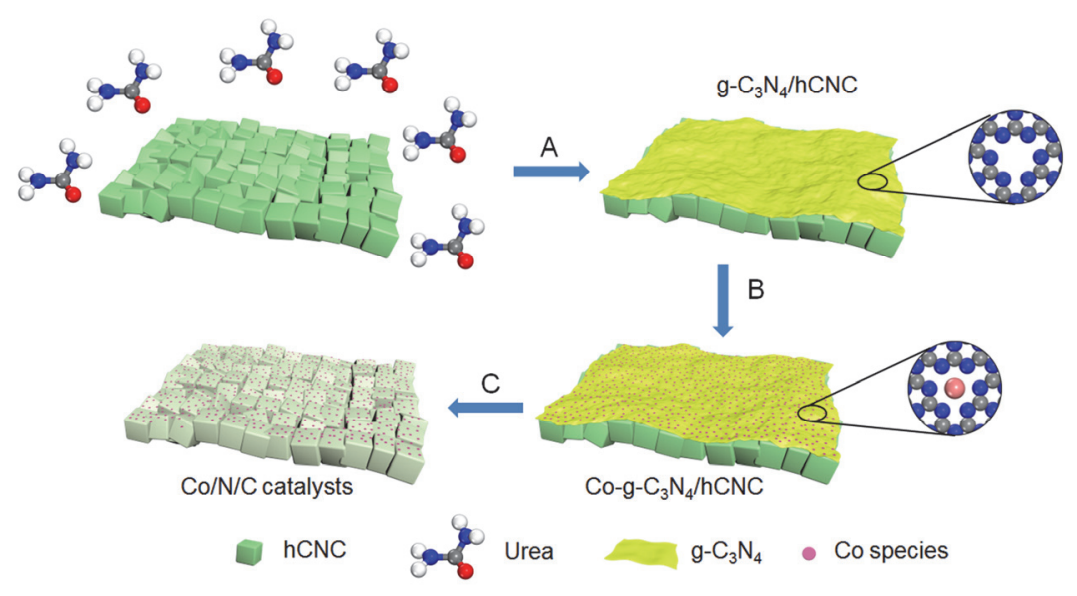

图 $1 \mathrm{Co} / \mathrm{N} / \mathrm{C}$ 催化剂的制备路线. (A) hCNC 与尿素浸渍混合并热解. (B) $\mathrm{Co}^{2+}$ 离子吸附与洗涤制备 $\mathrm{Co}-\mathrm{g}-\mathrm{C}_{3} \mathrm{~N}_{4} / \mathrm{hCNC}$ 复合物. (C) 热解.

Figure 1 Schematic illustration of the preparation of $\mathrm{Co} / \mathrm{N} / \mathrm{C}$ catalysts. (A) Mixing hCNC with urea by impregnation, and formation of $g-\mathrm{C}_{3} \mathrm{~N}_{4} / \mathrm{hCNC}$ by pyrolysis. (B) adsorption of $\mathrm{Co}^{2+}$ ions and washing. (C) pyrolysis and formation of $\mathrm{Co} / \mathrm{N} / \mathrm{C}$ catalysts 
$\mathrm{g}-\mathrm{C}_{3} \mathrm{~N}_{4}$ 类似的衍射峰(图 2c), 证实了 $\mathrm{g}-\mathrm{C}_{3} \mathrm{~N}_{4}$ 与 $\mathrm{hCNC}$ 的 复合. $X$ 射线光电子能谱 $(X P S)$ 测试显示 $g-C_{3} \mathrm{~N}_{4} / h C N C$ 复 合物中 $\mathrm{N}$ 含量为 25.38 at. $\%$ (图 $2 \mathrm{~d}$ ). $\mathrm{g}-\mathrm{C}_{3} \mathrm{~N}_{4} / \mathrm{hCNC}$ 复合 物的电导率为 $229 \mathrm{~S} \cdot \mathrm{m}^{-1}$, 略低于 $\mathrm{CNC}\left(395 \mathrm{~S} \cdot \mathrm{m}^{-1}\right)$, 但 远高于 $\mathrm{g}-\mathrm{C}_{3} \mathrm{~N}_{4}\left(2.6 \times 10^{-4} \mathrm{~S} \cdot \mathrm{m}^{-1}\right)$, 也表明 $\mathrm{g}-\mathrm{C}_{3} \mathrm{~N}_{4}$ 较均匀 地分散在 $\mathrm{hCNC}$ 表面. 吸附 $\mathrm{Co}^{2+}$ 离子之后, $\mathrm{Co}-\mathrm{g}-\mathrm{C}_{3} \mathrm{~N}_{4} /$ $\mathrm{hCNC}$ 复合物中 Co 含量为 0.41 at.\% (图 2d). 对比吸附 $\mathrm{Co}^{2+}$ 离子前后的 $\mathrm{N} 1 \mathrm{~s}$ 谱, 发现吸附 $\mathrm{Co}^{2+}$ 离子导致 $\mathrm{N} 1 \mathrm{~s}$ 峰向高结合能方向发生轻度偏移(图 S2), 表明 $\mathrm{N}$ 原子与 $\mathrm{Co}^{2+}$ 离子之间存在相互作用, 有利于 $\mathrm{Co}^{2+}$ 的高度分散. 用 $\mathrm{hCNC}$ 替代 $\mathrm{g}-\mathrm{C}_{3} \mathrm{~N}_{4} / \mathrm{hCNC}$ 复合物进行 $\mathrm{Co}^{2+}$ 离子吸附, 所得产物 $\mathrm{Co}-\mathrm{hCNC}$ 中没有检测到 $\mathrm{Co}$ 物种, 进一步说明 $\mathrm{g}-\mathrm{C}_{3} \mathrm{~N}_{4}$ 对 $\mathrm{Co}^{2+}$ 离子具有吸附作用(图 S3).

在高温下热处理 $\mathrm{Co}-\mathrm{g}-\mathrm{C}_{3} \mathrm{~N}_{4} / \mathrm{hCNC}$ 复合物获得 $\mathrm{Co} / \mathrm{N} / \mathrm{C}$ 催化剂, 其中 $800{ }^{\circ} \mathrm{C}$ 时所得产物的形貌与成分 表征示于图 3. 热处理产物由空心纳米笼组成, 跟 $\mathrm{hCNC}$ 类似，没有观察明显的颗粒存在，在笼壁外侧也没有看 到明显的颗粒状产物(图 3a, 3b), 这表明热处理过程中 大部分的 $\mathrm{g}-\mathrm{C}_{3} \mathrm{~N}_{4}$ 发生热分解, 残留的 $\mathrm{Co}$ 物种分散度高, 没有聚集. 热解产物的 XPS 谱(图 3c, 3d) 显示含有 C、N、 $\mathrm{Co} 、 \mathrm{O}$ 四种元素，其中 $\mathrm{Co} 、 \mathrm{~N}$ 的原子比分别为 0.31 at. \% 和 2.53 at. \%. 由于 $\mathrm{hCNC}$ 载体中只含 $\mathrm{C} 、 \mathrm{O}$ 两种元素(图 $2 \mathrm{~d}), \mathrm{Co} 、 \mathrm{~N}$ 物种来自 $\mathrm{g}-\mathrm{C}_{3} \mathrm{~N}_{4}$ 与 $\mathrm{Co}^{2+}$ 的热分解. 对 $\mathrm{N} 1 \mathrm{~s}$ 谱图进行拟合(图 3d), 可知催化剂中含有 5 种 $\mathrm{N}$ 物种, 其结合能分别位于 398.5、399.4、400.4、401.1 和 403.0 $\mathrm{eV}$ 处, 可分别归属为吡啶氮 $(\mathrm{N} 1), \mathrm{Co}-\mathrm{N}_{x}$ 物种(N2), 吡咯
氮(N3), 石墨氮(N4)和吡啶氮氧化物(N5) ${ }^{[31]}$, 该结果表 明热解所得的 $\mathrm{Co} / \mathrm{N} / \mathrm{C}$ 催化剂中形成了 $\mathrm{Co}-\mathrm{N}_{x}$ 活性物种. $\mathrm{Co} / \mathrm{N} / \mathrm{C}$ 催化剂的电导率为 $313 \mathrm{~S} \cdot \mathrm{m}^{-1}$, 保留了 $\mathrm{hCNC}$ 载 体的分级多孔结构(图 S4), 使其具有较大的电化学活性 面积 $\left(8.6 \mathrm{mF} \cdot \mathrm{cm}^{-2} \text { ) (图 } \mathrm{S} 5\right)^{[32]}$. 电化学阻抗谱表明, $\mathrm{Co} / \mathrm{N} / \mathrm{C}$ 催化剂的电荷转移电阻为 $1.2 \Omega$, 低于 $\mathrm{hCNC}$ 的 电荷转移电阻 $(2.0 \Omega$ ) (图 S6), 这可能与 $\mathrm{Co} 、 \mathrm{~N}$ 掺杂提高 了材料的浸润性有关. 低的电荷转移电阻表明 $\mathrm{Co} / \mathrm{N} / \mathrm{C}$ 催化剂更有利于电荷的界面传输 ${ }^{[33]}$, 从而有助于促进 其电极反应动力学过程.

使用旋转圆盘电极( RDE), 通过循环伏安 $(\mathrm{CV})$ 和线 性扫描伏安法(LSV)在 $0.1 \mathrm{~mol} \cdot \mathrm{L}^{-1} \mathrm{KOH}$ 电解液中表征 $\mathrm{Co} / \mathrm{N} / \mathrm{C}$ 催化剂的氧还原活性. 首先优化了 $\mathrm{Co} / \mathrm{N} / \mathrm{C}$ 催化 剂中 $\mathrm{Co}$ 负载量(图 S7). 对于 $\mathrm{Co}$ 含量分别为 $0.20,0.31$ 和 0.40 at. \% 的 $\mathrm{Co} / \mathrm{N} / \mathrm{C}$ 催化剂, 其 ORR 活性随 Co 含量 增加呈先增加后减小的趋势，在 Co 含量为 0.31 at.\% 时 最优. 在此基础上, 进一步考察了热处理温度对 ORR 活 性的影响, 如图 4 所示. 根据图 4a 中 CV 曲线可知, $\mathrm{Co} / \mathrm{N} / \mathrm{C}-\mathrm{T}$ 系列催化剂的峰电位随热处理温度升高呈先 增加后降低的趋势，依次为 $0.816 、 0.818 、 0.831$ 和 0.822 $\mathrm{V}$, 其中 $\mathrm{Co} / \mathrm{N} / \mathrm{C}-800$ 的峰电位达到最大值. 图 $4 \mathrm{~b}$ 中 $\mathrm{LSV}$ 曲线表现出跟 $\mathrm{CV}$ 曲线相同的变化规律, $\mathrm{Co} / \mathrm{N} / \mathrm{C}-800$ 催 化剂的起始电位和半波电位最高. 上述结果表明 $\mathrm{Co} / \mathrm{N} / \mathrm{C}-800$ 催化剂具有最佳的 ORR 活性, 在 $800{ }^{\circ} \mathrm{C}$ 下 热处理 $\mathrm{Co} / \mathrm{g}-\mathrm{C}_{3} \mathrm{~N}_{4} / \mathrm{hCNC}$ 复合物有利于形成 $\mathrm{Co}-\mathrm{N}_{x}$ 活性 物种, 从而展现出最优异的 ORR 活性 ${ }^{[34]}$.
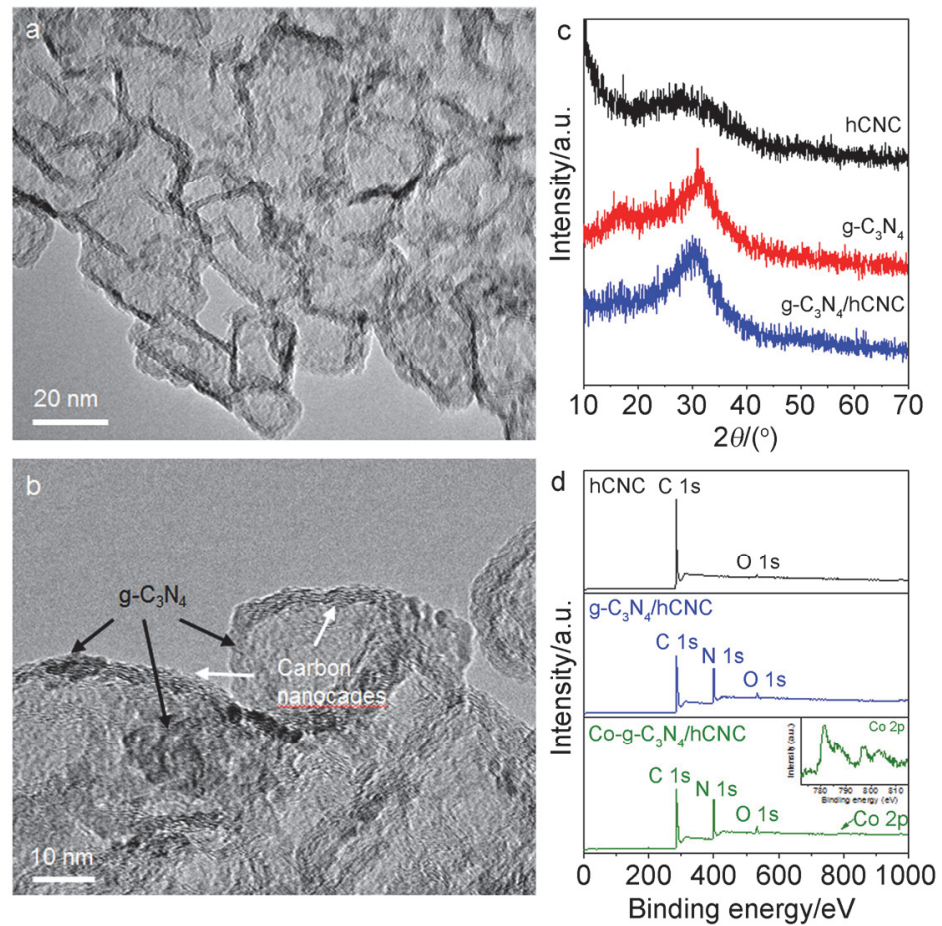

图 2 中间产物的形貌及结构表征. (a, b) g- $\mathrm{C}_{3} \mathrm{~N}_{4} / \mathrm{hCNC}$ 的(HR)TEM 照片. (c) XRD 谱. (d) hCNC、g- $\mathrm{C}_{3} \mathrm{~N}_{4} / \mathrm{hCNC}$ 和 Co-g-C $\mathrm{C}_{3} \mathrm{~N}_{4} / \mathrm{hCNC}$ 的 XPS 谱. Figure 2 Morphologic and structural characterizations of intermediates. (a, b) (HR)TEM images of $g-\mathrm{C}_{3} \mathrm{~N}_{4} / \mathrm{hCNC}$. (c) XRD patterns. (d) XPS spectra of $\mathrm{hCNC}, \mathrm{g}-\mathrm{C}_{3} \mathrm{~N}_{4} / \mathrm{hCNC}$ and $\mathrm{Co}-\mathrm{g}-\mathrm{C}_{3} \mathrm{~N}_{4} / \mathrm{hCNC}$. 

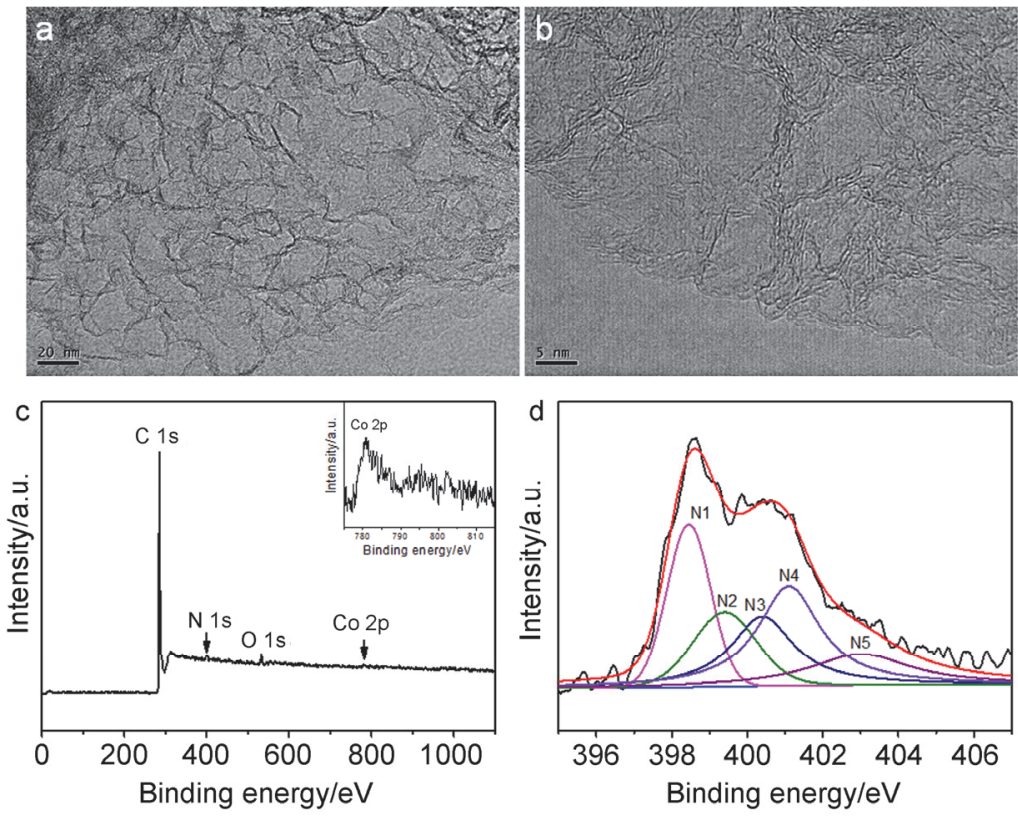

图 3 典型 $\mathrm{Co} / \mathrm{N} / \mathrm{C}$ 催化剂的形貌与成分. (a, b) (HR)TEM 照片. (c) XPS 全谱, 插图为 Co $2 p$ 谱. (d) N 1s 谱

Figure 3 Morphology and composition of typical Co/N/C catalyst. (a, b) (HR)TEM images. (c) XPS spectra. Inset is the Co 2p spectrum. (d) N 1s spectrum
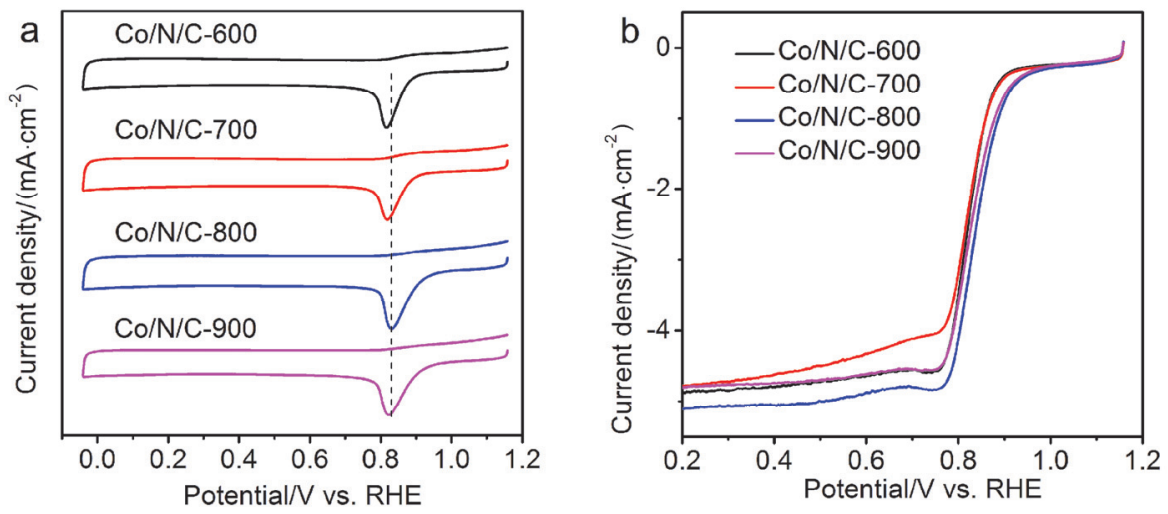

图 $4 \mathrm{Co} / \mathrm{N} / \mathrm{C}-\mathrm{T}$ 系列催化剂在 $0.1 \mathrm{~mol} \cdot \mathrm{L}^{-1} \mathrm{KOH}$ 电解液中的 ORR 性能. (a) $\mathrm{CV}$ 曲线. (b) LSV 曲线.

Figure 4 ORR performance of $\mathrm{Co} / \mathrm{N} / \mathrm{C}-\mathrm{T}$ catalysts in $0.1 \mathrm{~mol} \cdot \mathrm{L}^{-1} \mathrm{KOH}$ solution. (a) $\mathrm{CV}$ curves. (b) LSV curves

图 5 为 $\mathrm{Co} / \mathrm{N} / \mathrm{C}-800$ 催化剂与 $\mathrm{hCNC}$ 载体、商业 $\mathrm{Pt} / \mathrm{C}$ 的 ORR 性能比较. 在 $\mathrm{N}_{2}$ 饱和的电解液中, $\mathrm{Co} / \mathrm{N} / \mathrm{C}-800$ 催化剂和 $\mathrm{hCNC}$ 载体的 $\mathrm{CV}$ 曲线没有明显的特征峰; 在 $\mathrm{O}_{2}$ 饱和的电解液中, $\mathrm{Co} / \mathrm{N} / \mathrm{C}-800$ 催化剂的峰电位为 $0.831 \mathrm{~V}$, 高于 $\mathrm{hCNC}$ 的峰电位 (约 $0.741 \mathrm{~V}$ ) (图 5a), 表明 前者的 ORR 活性显著优于 $\mathrm{hCNC}$ 载体, 该活性源于吸 附了 $\mathrm{Co}^{2+}$ 的 $\mathrm{g}-\mathrm{C}_{3} \mathrm{~N}_{4}$ 的热解产物. 如将催化剂在 $\mathrm{N}_{2}$ 和 $\mathrm{O}_{2}$ 饱和的电解液中 CV 和 LSV 曲线分离点的电位定义为起 始电位(图 5a, 图 S8), Co/N/C-800 催化剂的起始电位为 $0.97 \mathrm{~V}$, 同样高于 $\mathrm{hCNC}$ 的 $0.83 \mathrm{~V}$. 对比 LSV 曲线(图 5b), 可看到 $\mathrm{Co} / \mathrm{N} / \mathrm{C}$ 催化剂的半波电位与 $\mathrm{Pt} / \mathrm{C}$ 相当, 在电位 为 $0.9 \mathrm{~V}$ (vs. RHE) 时基于金属物种的质量活性是商业 $\mathrm{Pt} / \mathrm{C}$ 催化剂 $(20 \mathrm{wt} \% \mathrm{Pt}$ ) 的 5.72 倍(图 S9), 其极限电流 甚至稍高于商业 $\mathrm{Pt} / \mathrm{C}$ 催化剂, 表明其具有优异的 ORR 活性. 跟文献报道的钴基催化剂相比, 我们制备的
$\mathrm{Co} / \mathrm{N} / \mathrm{C}$ 催化剂的 ORR 性能处于先进水平(表 $\mathrm{S} 1$ ). 旋转 圆盘圆环电极(RRDE)测试计算得到 $\mathrm{Co} / \mathrm{N} / \mathrm{C}-800$ 催化剂 的电子转移数 $(n)$ 为约 $3.65 \pm 0.10$, 双氧水 $\left(\mathrm{HO}_{2}{ }^{-}\right)$平均产 率为约 18.1\% (图 5c, 5d), 表明在该催化剂作用下, ORR 反应按照 4 电子过程为主的反应历程进行.

良好的稳定性及抗甲醇干扰性能也是 ORR 催化剂 的重要指标. 采用计时电流法表征 $\mathrm{Co} / \mathrm{N} / \mathrm{C}-800$ 催化剂 的 ORR 稳定性, 结果如图 $5 \mathrm{e}, 5 \mathrm{f}$ 所示. 经过 $25 \mathrm{~h}$ 连续测 试后, $\mathrm{Co} / \mathrm{N} / \mathrm{C}-800$ 催化剂的电流密度下降了约 $30 \%$, 明 显低于商业 $\mathrm{Pt} / \mathrm{C}$ 的 $52 \%$, 表明 $\mathrm{Co} / \mathrm{N} / \mathrm{C}-800$ 催化剂的稳 定性优于商业 $\mathrm{Pt} / \mathrm{C}$. 此外，甲醇渗透模拟实验显示，当 甲醇加入反应系统后, $\mathrm{Co} / \mathrm{N} / \mathrm{C}-800$ 催化剂的电流基本无 变化, 具有优异的抗甲醇干扰的性能(图 5f), 显著优于 $\mathrm{Pt} / \mathrm{C}$ 催化剂. 这表明 $\mathrm{Co} / \mathrm{N} / \mathrm{C}$ 催化剂用作直接甲醇燃料 电池的 ORR 催化剂时更有优势. 

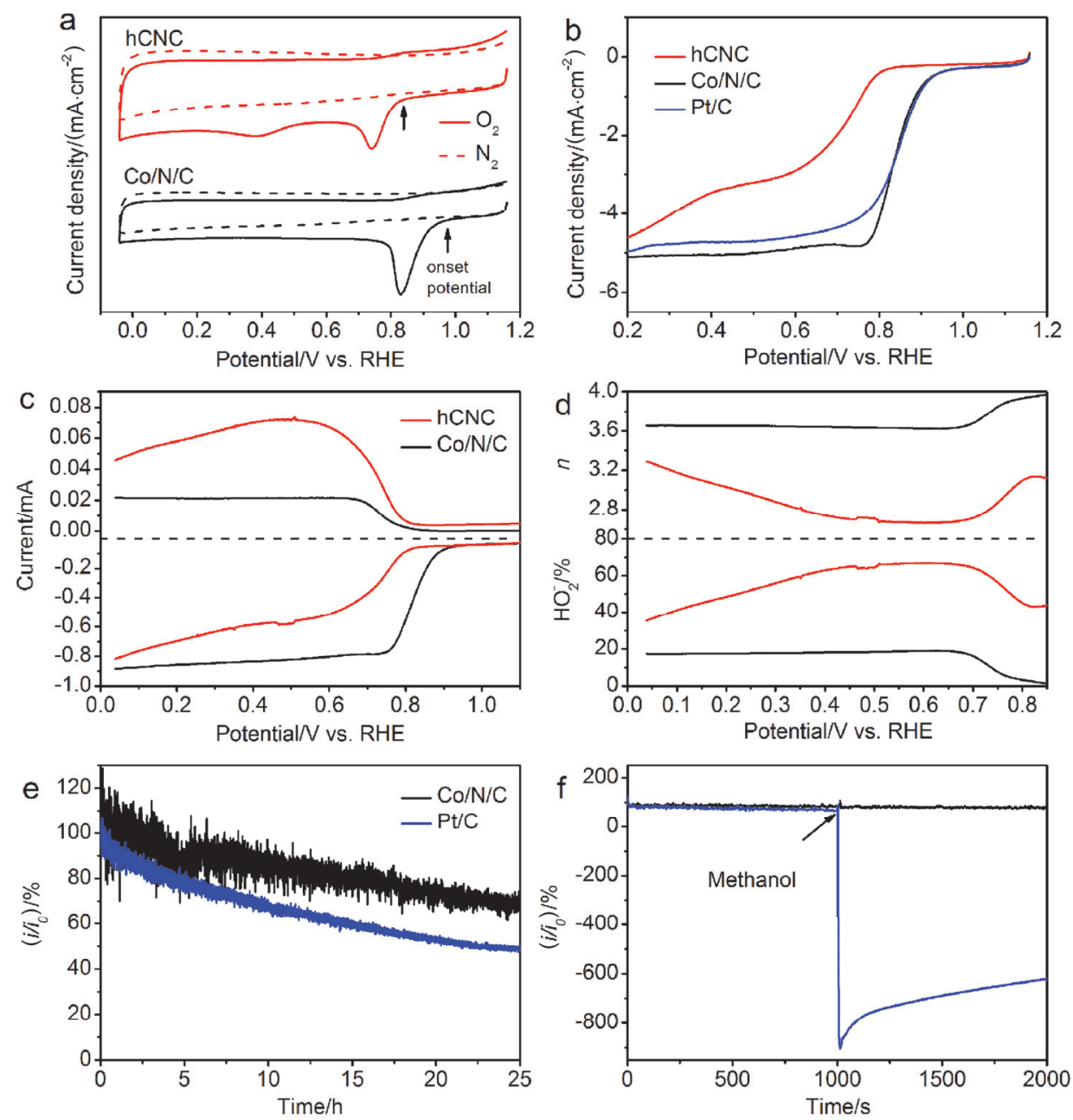

图 $5 \mathrm{Co} / \mathrm{N} / \mathrm{C}$ 催化剂、 $\mathrm{hCNC}$ 与商业 $\mathrm{Pt} / \mathrm{C}$ 的 $\mathrm{ORR}$ 性能比较. (a) 在 $\mathrm{O}_{2}\left(-\right.$ ) 和 $\mathrm{N}_{2}(---)$ 饱和的电解液中测得的 $\mathrm{CV}$ 曲线. (b) LSV 曲线. (c) RRDE 曲线. (d) 电子转移数和 $\mathrm{HO}_{2}{ }^{-}$产率的变化曲线. (e) $25 \mathrm{~h}$ 内 $i-t$ 曲线. (f) 甲醇渗透实验的 $i-t$ 曲线, $1000 \mathrm{~s}$ 时加入 $2 \mathrm{vol} \%$ 的甲醇.

Figure 5 ORR performance of $\mathrm{Co} / \mathrm{N} / \mathrm{C}, \mathrm{hCNC}$ and commercial $\mathrm{Pt} / \mathrm{C}$ in $0.1 \mathrm{~mol} \cdot \mathrm{L}^{-1} \mathrm{KOH}$ solution. (a) $\mathrm{CV}$ curves in $\mathrm{N}_{2}$-saturated (---) and $\mathrm{O}_{2}$-saturated (-) electrolytes. (b) LSV curves. (c) RRDE curves. (d) electron transfer number (n) and $\mathrm{HO}_{2}{ }^{-}$yield vs potential. (e) stability tests. (f) methanol crossover tests of $\mathrm{Co} / \mathrm{N} / \mathrm{C}-800$ and Pt/C. $2 \%(V / V)$ methanol was added to the electrochemical cell at $1000 \mathrm{~s}$ as shown by the arrow.

\section{3 结论}

将 $\mathrm{g}-\mathrm{C}_{3} \mathrm{~N}_{4}$ 负载于高导电性 $\mathrm{hCNC}$ 表面生成 g- $\mathrm{C}_{3} \mathrm{~N}_{4} / \mathrm{hCNC}$ 复合物, 进而利用表层 $\mathrm{g}-\mathrm{C}_{3} \mathrm{~N}_{4}$ 的配位和限 域作用针定大量 $\mathrm{Co}^{2+}$ 离子, 经热解制得了活性位高度 暴露、导电性好、孔结构丰富的 $\mathrm{Co} / \mathrm{N} / \mathrm{C}$ 催化剂. 在碱 性介质中, $800{ }^{\circ} \mathrm{C}$ 热解得到的最优催化剂展现出优异 ORR 活性, 其 CV 峰电位为 $0.831 \mathrm{~V}, \mathrm{LSV}$ 曲线的半波电 位与商业 $\mathrm{Pt} / \mathrm{C}$ 催化剂相当, 且抗甲醇干扰性能和稳定性 优异. 此项研究有效解决了 $\mathrm{M} / \mathrm{N} / \mathrm{C}$ 催化剂的活性位容 易被包埋的缺点, 提供了一种构建具有高度暴露活性位 的 $\mathrm{M} / \mathrm{N} / \mathrm{C}$ 催化剂的有效策略.

\section{4 实验部分}

\section{1 材料制备}

$\mathrm{hCNC}$ 的制备: 采用本课题组发展的原位 $\mathrm{MgO}$ 模 板法制备 $\mathrm{hCNC}{ }^{[24]}$.
$\mathrm{g}-\mathrm{C}_{3} \mathrm{~N}_{4} / \mathrm{hCNC}$ 复合物的制备: (1) 称取 $2 \mathrm{~g}$ 尿素溶于 $60 \mathrm{~mL}$ 蒸馏水中, 再加入 $100 \mathrm{mg} \mathrm{hCNC}$ 进行超声分散, 加热到 $80{ }^{\circ} \mathrm{C}$, 摚拌下蒸干溶剂; (2) 得到的混合物置于 管式炉, $\mathrm{N}_{2}$ 气氛中于 $550{ }^{\circ} \mathrm{C}$ 热处理 $2 \mathrm{~h}$, 冷却后得到 g- $\mathrm{C}_{3} \mathrm{~N}_{4} / \mathrm{hCNC}$ 复合物.

$\mathrm{Co} / \mathrm{N} / \mathrm{C}$ 催化剂的制备: (1) 称取 $50 \mathrm{mg} \mathrm{g-} \mathrm{C}_{3} \mathrm{~N}_{4} /$ $\mathrm{hCNC}$ 分散于 $60 \mathrm{~mL}$ 蒸馏水中, 加入一定量的氯化亚钴 $\left(\mathrm{CoCl}_{2}\right)$ 溶液, 加热到 $70{ }^{\circ} \mathrm{C}$, 搅拌 $10 \mathrm{~h}$; (2) 过滤, 用乙 醇、水多次洗涤, $70{ }^{\circ} \mathrm{C}$ 烘干; (3) 混合物在 $\mathrm{N}_{2}$ 气氛中于 不同温度 $\left(600 \sim 900{ }^{\circ} \mathrm{C}\right)$ 热处理 $2 \mathrm{~h}$, 所得产物被命名为 $\mathrm{Co} / \mathrm{N} / \mathrm{C}-T$ ( $T$ 为 $600,700,800,900$, 对应于热处理温度).

\section{2 材料表征}

采用扫描电子显微镜(SEM, Hitachi S4800, $10 \mathrm{kV}$ ) 和高分辨透射电子显微镜(HRTEM, JEM2100, $200 \mathrm{kV}$, 搭载 EDS, ANTAGE DS(EDS)NORON)观察样品形貌; 采用 X 射线衍射仪 (XRD, D8 ADVANCE, Co 靶, 工作电 
压 $40 \mathrm{kV}$, 电流 $35 \mathrm{~mA}$ )表征其晶体结构; 采用 $\mathrm{X}$ 射线光 电子能谱仪(XPS, Thermo ESCALAB 250)表征元素的含 量和化学状态, 光源为单色 $\mathrm{Al} \mathrm{K \alpha}(h v=1486.6 \mathrm{eV})$; 采 用 Thermo SCIENTIFIC SURFUR 测定 hCNC 的比表面 积及孔分布, 样品在 $300{ }^{\circ} \mathrm{C}$ 脱气活化 $4 \mathrm{~h}$, 然后采用容 量法以 $\mathrm{N}_{2}$ 为吸附质在 $77 \mathrm{~K}$ 下进行测试. 采用 BET (Brunauer-Emmett-Teller)方法计算比表面积, 采用 BJH (Barrett-Joyner-Halenda)方法得到孔径分布数据.

\section{3 电化学性能测试}

采用三电极系统进行电化学测试, 以滴涂了催化剂 的旋转圆盘电极为工作电极、银/氯化银 $(\mathrm{Ag} / \mathrm{AgCl})$ 为参 比电极、石墨电极为对电极. 工作电极的制备过程如下: 玻碳电极依次用 $1.0 、 0.3 、 0.05 \mu \mathrm{m}$ 的 $\mathrm{Al}_{2} \mathrm{O}_{3}$ 粉抛光, 用 二次去离子水清洗, 室温下晾干. 将 $2.0 \mathrm{mg}$ 催化剂超声 分散到 $0.8 \mathrm{~mL}$ 水、 $0.2 \mathrm{~mL}$ 乙醇和 $40 \mu \mathrm{L}$ Nafion(Dupont, $5 \%$ )的混合溶液中, 超声 $1 \mathrm{~h}$. 取 $20 \mu \mathrm{L}$ 上述悬浮液滴到 抛光后的玻碳电极表面, 室温下晾干 $12 \mathrm{~h}$, 备用. 玻碳 电极上催化剂的负载量为 $0.20 \mathrm{mg} \cdot \mathrm{cm}^{-2}$.

所有电化学性能测试均在 CHI $760 \mathrm{C}$ 电化学工作站 上完成, 测试温度为 $25{ }^{\circ} \mathrm{C}$, 获得循环伏安曲线(cyclic voltammetry, CV)、基于旋转圆盘电极 (rotating disk electrode, RDE)和旋转圆盘圆环电极(rotating ring-disk electrode, RRDE)的线性扫描伏安曲线(LSV)和电流-时 间曲线 $(i-t) . \mathrm{CV} 、 \mathrm{RDE}$ 和 $\mathrm{RRDE}$ 测试均在 $0.1 \mathrm{~mol} \cdot \mathrm{L}^{-1}$ $\mathrm{KOH}$ 中进行, 扫描速度均为 $10 \mathrm{mV} \cdot \mathrm{s}^{-1}, \mathrm{RDE}$ 和 RRDE 电极的旋转速度为 $1600 \mathrm{r} / \mathrm{min}$. 稳定性测试的偏电压均 为 $-0.3 \mathrm{~V}$ (vs. $\mathrm{Ag} / \mathrm{AgCl}$ ), 抗甲醇测试中 $\mathrm{Co} / \mathrm{N} / \mathrm{C}$ 样品的 偏电压为 $-0.3 \mathrm{~V}$ (vs. $\mathrm{Ag} / \mathrm{AgCl}), \mathrm{Pt} / \mathrm{C}$ 的偏电压为 $-0.3 \mathrm{~V}$ (vs. $\mathrm{Ag} / \mathrm{AgCl}), \mathrm{O}_{2}$ 通入流量为 $15 \mathrm{~mL} \cdot \mathrm{min}^{-1}$. ORR 过程中 电子转移数 $(n)$ 和 $\mathrm{HO}_{2}{ }^{-}$产率分别用公式 $n=4 I_{\text {disk }} /\left(I_{\text {disk }}+\right.$ $\left.I_{\text {ring }} / N\right)$ 和 $\mathrm{HO}_{2}{ }^{-} \%=2\left(I_{\text {ring }} / N\right) /\left(I_{\text {disk }}+\left(I_{\text {ring }} / N\right)\right) \times 100 \%$ 计算 得到, $I_{\text {disk }}$ 和 $I_{\text {ring }}$ 分别为 RRDE 测试中的盘电流和环电流, $N$ 是环电极的收集系数 $(N=0.26)$, 环电极的偏电压为 $0.5 \mathrm{~V}$ (vs. $\mathrm{Ag} / \mathrm{AgCl}$ ).

\section{References}

[1] Gewirth, A. A.; Thorum, M. S. Inorg. Chem. 2010, 49, 3557.

[2] Zhong, C.-J.; Luo, J.; Njoki, P. N.; Mott, D.; Wanjala, B.; Loukrakpam, R.; Lim, S.; Wang, L.; Fang, B.; Xu, Z. Energy Environ. Sci. 2008, 1,454 .
[3] Debe, M. K. Nature 2012, 486, 43.

[4] Gasteiger, H. A.; Marković, N. M. Science 2009, 324, 48.

[5] Zhong, G.; Wang, H.; Yu, H.; Peng, F. Acta Chim. Sinica 2017, 75, 943 (in Chinese). (钟国玉，王红娟，余皓，彭峰，化学学报, 2017 , 75,943 .)

[6] Sun, T.; Wu, Q.; Zhuo, O.; Jiang, Y.; Bu, Y.; Yang, L.; Wang, X.; Hu, Z. Nanoscale 2016, 8,8480 .

[7] Wu, G.; More, K. L.; Johnston, C. M.; Zelenay, P. Science 2011, 332, 443.

[8] Xiao, M.; Zhu, J.; Feng, L.; Liu, C.; Xing, W. Adv. Mater. 2015, 27, 2521.

[9] Wu, G.; Zelenay, P. Acc. Chem. Res. 2013, 46, 1878.

[10] Antolini, E. Appl. Catal., B: Environ. 2009, 88, 1.

[11] Liang, J.; Zhou, R. F.; Chen, X. M.; Tang, Y. H.; Qiao, S. Z. Adv. Mater. 2014, 26, 6074.

[12] Yue, B.; Ma, Y.; Tao, H.; Yu, L.; Jian, G.; Wang, X.; Wang, X.; Lu, Y.; Hu, Z. J. Mater. Chem. 2008, 18, 1747.

[13] Feng, H.; Ma, J.; Hu, Z. J. Mater. Chem. 2010, 20, 1702.

[14] Zheng, Y.; Jiao, Y.; Zhu, Y.; Cai, Q.; Vasileff, A.; Li, L. H.; Han, Y.; Chen, Y.; Qiao, S.-Z. J. Am. Chem. Soc. 2017, 139, 3336.

[15] Qing, H.; Zhihua, C.; Jian, G.; Yang, Z.; Zhipan, Z.; Liming, D.; Liangti, Q. Adv. Funct. Mater. 2017, 27, 1606352.

[16] Vilé, G.; Albani, D.; Nachtegaal, M.; Chen, Z.; Dontsova, D.; Antonietti, M.; López, N.; Pérez-Ramírez, J. Angew. Chem. Int. Ed. 2015, $54,11265$.

[17] Li, X.; Bi, W.; Zhang, L.; Tao, S.; Chu, W.; Zhang, Q.; Luo, Y.; Wu, C.; Xie, Y. Adv. Mater. 2016, 28, 2427.

[18] Liu, Q.; Zhang, J. Langmuir 2013, 29, 3821.

[19] Yu, Q.; Xu, J.; Wu, C.; Guan, L. RSC Adv. 2015, 5, 65303.

[20] Zheng, Y.; Jiao, Y.; Zhu, Y.; Li, L. H.; Han, Y.; Chen, Y.; Du, A.; Jaroniec, M.; Qiao, S. Z. Nat. Commun. 2014, 5, 3783.

[21] Duan, J.; Chen, S.; Jaroniec, M.; Qiao, S. Z. ACS Nano 2015, 9, 931.

[22] Ji, L.; Yao, Z.; Jun, C.; Jian, L.; Denisa, H.-J.; Mietek, J.; Zhang, Q. S. Angew. Chem. Int. Ed. 2012, 51, 3892.

[23] Zheng, Y.; Jiao, Y.; Chen, J.; Liu, J.; Liang, J.; Du, A.; Zhang, W.; Zhu, Z.; Smith, S. C.; Jaroniec, M.; Lu, G. Q.; Qiao, S. Z. J. Am. Chem. Soc. 2011, 133, 20116.

[24] Wu, Q.; Yang, L.; Wang, X.; Hu, Z. Acc. Chem. Res. 2017, 50, 435.

[25] Sun, T.; Wu, Q.; Che, R.; Bu, Y.; Jiang, Y.; Li, Y.; Yang, L.; Wang, X.; Hu, Z. ACS Catal. 2015, 5, 1857.

[26] Lyu, Z.; Xu, D.; Yang, L.; Che, R.; Feng, R.; Zhao, J.; Li, Y.; Wu, Q.; Wang, X.; Hu, Z. Nano Energy 2015, 12, 657.

[27] Bu, Y.; Sun, T.; Cai, Y.; Du, L.; Zhuo, O.; Yang, L.; Wu, Q.; Wang, X.; Hu, Z. Adv. Mater. 2017, 29, 1700470.

[28] Wang, L. W.; Feng, R.; Xia, J. Z.; Chen, S.; Wu, Q.; Yang, L. J.; Wang, X. Z.; Hu, Z. Acta Chim. Sinica 2014, 72, 1070 (in Chinese). (王立伟, 冯瑞, 夏婧竹, 陈盛, 吴强, 杨立军, 王喜章, 胡征, 化 学学报, 2014, 72, 1070.)

[29] Wang, X.; Chen, X.; Thomas, A.; Fu, X.; Antonietti, M. Adv. Mater. 2009, 21, 1609 .

[30] Zou, X.; Su, J.; Silva, R.; Goswami, A.; Sathe, B. R.; Asefa, T. Chem. Commun. 2013, 49, 7522.

[31] Niu, K.; Yang, B.; Cui, J.; Jin, J.; Fu, X.; Zhao, Q.; Zhang, J. J. Power Sources 2013, 243, 65.

[32] Kuang, M.; Wang, Q.; Han, P.; Zheng, G. Adv. Energy Mater. 2017, 7, 1700193 .

[33] Zhao, J.; Lai, H.; Lyu, Z.; Jiang, Y.; Xie, K.; Wang, X.; Wu, Q.; Yang, L.; Jin, Z.; Ma, Y.; Liu, J.; Hu, Z. Adv. Mater. 2015, 27, 3541.

[34] Fei, H.; Dong, J.; Arellano-Jiménez, M. J.; Ye, G.; Kim, N. D.; Samuel, E. L. G.; Peng, Z.; Zhu, Z.; Qin, F.; Bao, J.; Yacaman, M. J.; Ajayan, P. M.; Chen, D.; Tour, J. M. Nat. Commun. 2015, 6, 8668. 